\title{
Active tuberculosis case-finding among drug users and homeless persons: after the outbreak
}

\begin{abstract}
To the Editor:
Tuberculosis (TB) control in high-risk settings and populations is a fascinating challenge, as it also is in the context of the World Health Organization (WHO) End TB strategy [1]; and it is frequently reported in medical journals. We previously described how an outbreak was built up among illicit drug users and homeless persons in Rotterdam, the Netherlands, and successfully controlled by a systematic targeted TB active case-finding programme, using a mobile digital X-ray unit (MDXU) [2,3]. On the contrast, programmatic implications after an outbreak are rarely reported. This study evaluates the intervention among these urban risk groups by describing trends of TB disease, recent transmission, active case finding and treatment outcome, and by comparing efficiency and yield of screening during outbreak management (May 2002-2005) with post-outbreak screening (2006-2014).
\end{abstract}

TB case and DNA fingerprint based recent transmission definitions were previously described [3]. All notified TB cases among illicit drug users with a registered address in Rotterdam and homeless persons residing in Rotterdam, diagnosed between May 1, 2002, and December 31, 2014, were included. The efficiency of the active case-finding programme is expressed as the number needed to screen (NNS) to identify one TB case. Yield is calculated as the proportion of TB cases identified through the active case-finding programme relative to the total TB caseload for the risk groups involved [4].

During outbreak management, all 14 facilities for illicit drug users and homeless persons in Rotterdam were visited twice per year but this number gradually reduced during the post-outbreak screening period because some major facilities were closed, such as the street prostitution zone. Screening in some other locations was stopped because TB occurred only incidentally or not at all among their residents or clients. By the end of 2014, screening still took place in three facilities. The annual numbers of radiographs made in the active case-finding programme was on average 2500 during outbreak management, but reduced from 1951 in 2006 to 366 in 2014.

The annual number of notified TB cases among illicit drug users and homeless persons in Rotterdam declined from an average of 19 cases (71 in 3.7 years) during outbreak management to an average of five cases (42 in 9 years) during post-outbreak management (table 1). The proportion of illicit drug users or homeless persons with a Mycobacterium tuberculosis strain attributed to recent transmission was $>80 \%$ when the outbreak built up, reduced to $45 \%$ at the end of the outbreak [4] and was, on average, $35 \%$ during the post-outbreak screening period, with $0 \%$ in 2010, 2012, 2013 and 2014. During the outbreak management period, 12 of the 28 TB cases (42.9\%) identified by the MDXU TB screening programme had a microscopy-positive sputum smear and 27 (96.4\%) cases were culture-confirmed. In the same period, another seven TB cases among illicit drug users or homeless persons were identified through contact investigation and seven via TB screening in prisons, resulting in 42 (59.2\%) of $71 \mathrm{~TB}$ cases among these risk groups found through active case-finding. During the post-outbreak screening period, none of the four TB cases identified by the MDXU TB screening programme had a microscopy-positive sputum smear; two cases were culture confirmed. In the same period, four TB cases were identified through the TB screening programme in prisons and one case otherwise, resulting in nine $(21.4 \%)$ of $42 \mathrm{~TB}$ cases among these risk groups found through active case finding.

Of the 32 cases found through the MDXU TB screening programme, 91\% completed treatment, 3\% died (one case, not due to TB) and 6\% (two cases) were lost to follow-up. The efficiency of the programme reduced from 325 to 2180 during the two periods and the yield among the target population declined from $39.4 \%$ to $9.5 \%$.

WHO published guidance on principles for systematic screening for active tuberculosis (TB) [5]. Elsewhere Golub and Dowdy [4] discussed the methodological challenges in implementation and evaluation. A challenge of active case-finding is to have high-specificity strategies that detect cases early in the disease spectrum and avert disease burden and transmission, which are affordable [4]. Digital radiographic screening can achieve a high level of specificity and accuracy in an operational setting [6,7]. During both study periods in Rotterdam, over $88 \%$ of all TB cases actively identified were culture confirmed. None of the cases detected by the screening programme post-outbreak management was infectious, indicating early case finding. Another challenge is the selection of the appropriate risk groups (and being able to involve 
TABLE 1 Number of tuberculosis (TB) cases in Rotterdam May 2002-2014

$\begin{array}{cc}\begin{array}{c}\text { TB cases in } \\ \text { Rotterdam }\end{array} & \begin{array}{c}\text { Homeless/illicit } \\ \text { drug-using TB } \\ \text { (a) }\end{array} \\ & \begin{array}{c}\text { cases in } \\ \text { Rotterdam (b) }\end{array}\end{array}$

Proportion
homeless/illicit
drug-using TB
cases (b/a)

TB cases
identified by
MDXU TB
screening (c)

\begin{tabular}{|c|c|c|}
\hline $\begin{array}{l}\text { Number of } \\
\text { screenings } \\
\text { (d) }\end{array}$ & $\begin{array}{l}\text { Number } \\
\text { needed to } \\
\text { screen } \\
\text { (d/c) }\end{array}$ & $\begin{array}{c}\text { Yield lof } \\
\text { target } \\
\text { population] } \\
\text { [c/b] }\end{array}$ \\
\hline
\end{tabular}

\begin{tabular}{|c|c|c|c|c|c|c|c|}
\hline May-December 2002 & 100 & 17 & $17.0 \%$ & 11 & 1615 & & \\
\hline 2003 & 171 & 25 & $14.6 \%$ & 10 & 2789 & & \\
\hline 2004 & 164 & 18 & $11.0 \%$ & 1 & 2430 & & \\
\hline 2005 & 130 & 11 & $8.5 \%$ & 6 & 2265 & & \\
\hline Outbreak management & 565 & 71 & $12.6 \%$ & 28 & 9099 & 325 & $39.4 \%$ \\
\hline 2006 & 127 & 5 & $3.9 \%$ & 2 & 1951 & & \\
\hline 2007 & 109 & 4 & $3.7 \%$ & 0 & 1567 & & \\
\hline 2008 & 100 & 10 & $10.0 \%$ & 1 & 1308 & & \\
\hline 2009 & 129 & 6 & $4.7 \%$ & 0 & 1231 & & \\
\hline 2010 & 110 & 5 & $4.5 \%$ & 1 & 839 & & \\
\hline 2011 & 89 & 4 & $4.5 \%$ & 0 & 814 & & \\
\hline 2012 & 98 & 4 & $4.1 \%$ & 0 & 370 & & \\
\hline 2013 & 81 & 2 & $2.5 \%$ & 0 & 272 & & \\
\hline 2014 & 68 & 2 & $2.9 \%$ & 0 & 366 & & \\
\hline Post-outbreak period & 911 & 42 & $4.6 \%$ & 4 & 8718 & 2180 & $9.5 \%$ \\
\hline
\end{tabular}

Annual number of all homeless persons and illicit drug users notified with TB in Rotterdam, annual proportion of homeless or drug using TB cases relative to the total caseload, annual number of homeless or drug using TB cases identified by the mobile digital X-ray Unit (MDXU), annual number of screenings, annual efficiency (number needed to screen) and annual yield (proportion of homeless persons and illicit drug users identified by the MDXU relative to the total number of homeless or drug using TB cases (target population) of the intervention.

these often hard-to-reach individuals in the screening and follow-up), and integration with the broader health and social care systems [4]. Pre-outbreak management, high TB incidence rates, high proportions contributing to the total TB caseload, and DNA fingerprint cluster analysis indicated on-going transmission among illicit drug users and homeless persons in Rotterdam, confirmed during outbreak management [2,3]. During the outbreak, the MDXU screening programme reached about two-thirds (63\%) of the estimated target population at least once per year, and $23 \%$, possibly the resident risk group members, at least twice per year [8]. The active case-finding programme in Rotterdam was embedded in the Public Health TB Clinic activities and implemented during a comprehensive social rehabilitation programme for illicit drug users and homeless persons, funded by the city of Rotterdam [9]. A further challenge is to find an optimal approach towards who should be screened, how and at what intervals [4]. We defined a target population using services for illicit drug users and homeless persons, implemented radiographic MDXU screening for active intrathoracic $\mathrm{TB}$, but did not screen for latent $\mathrm{TB}$ infection (LTBI), and applied an opting-out selection approach, i.e. (strong) persuasion for participation by in-service social workers and paramedical staff, during bi-annual visits. Similar strategies have been employed among illicit drug users and homeless persons in other Western European urban conglomerates $[6,10,11]$. The final challenge is that TB screening should result in reduced morbidity and mortality on individual level and reduced transmission as public health effect [4]. The impact of the radiographic screening programme in Rotterdam was a decline of number of TB cases among illicit drug users and homeless persons, low mortality in the target population with one case dying of TB since 2002, and reduced recent transmission, also affecting the larger population.

Screening for active TB in selected high-risk groups should include careful case-based surveillance that allows for monitoring and evaluation of the screening strategy and can guide reprioritisation and discontinuation of screening when the yield reaches a low level [12]. Criteria for scaling down can be similar to criteria used to start active case-finding interventions among risk groups targeted, such as trend of TB cases, proportion of TB cases relative to the total caseload and incidence rates, as well as DNA fingerprint cluster analysis. Criteria can be (partly) related to the active case-finding intervention, such as the efficiency (NNS or prevalence rate) and yield, or based on molecular epidemiology, e.g. the trend of assumed recent transmission in the risk group.

The action framework for low-incidence countries towards TB elimination mentions TB control and prevention among vulnerable and hard-to-reach groups as a priority action area [12]. As incidence decreases, TB becomes more and more concentrated in certain vulnerable groups, with overlapping risk factors, not only having a greater risk of developing TB but also for not early accessing diagnostic services and not adhering to TB treatment. A complementary intervention to radiographic screening for active intrathoracic 
TB that may be considered among illicit drug users and homeless persons in the context of TB elimination is targeted screening for those with LTBI, the pool of future TB cases, with the intention to offer preventive treatment, although challenges, with the currently recommended treatment regimens, are ample and the evidence for epidemiological impact is weak [13-15]. We decided not to continue with LTBI screening since the annual number of TB cases among illicit drug users and homeless persons in Rotterdam was low and in the last 5 years of the screening programme evidence of recent transmission was virtually absent.

In Rotterdam, after an outbreak, a combination of public health and social rehabilitation interventions had considerable impact on TB incidence and recent transmission among illicit drug users and homeless persons. Efficiency and yield of the active case-finding programme substantially reduced. We conclude that when alternative active case-finding interventions are in place, such as prison screening or contact investigation, the latter shifting priority to LTBI, and in particular when low-threshold TB services for self-reporting or referral are maintained, targeted radiological TB screening among urban TB risk groups can be scaled down or stopped completely, as was done on January 1, 2015 in Rotterdam.

@ERSpublications

Efficiency and yield of an active TB case-finding programme during and after an outbreak can guide scaling down http://ow.ly/ZT1Me

Rob van Hest ${ }^{1,2}$ and Gerard de Vries ${ }^{3}$

${ }^{1}$ Municipal Public Health Service Rotterdam-Rijnmond, Rotterdam, The Netherlands. ${ }^{2}$ Municipal Public Health Service Groningen, Groningen, The Netherlands. ${ }^{3} \mathrm{KNCV}$ Tuberculosis Foundation, The Hague, The Netherlands.

Correspondence: Rob van Hest, Tuberculosis Control Department, Municipal Public Health Service RotterdamRijnmond, P.O. Box 70032, 3000 LP Rotterdam, The Netherlands. E-mail: nah.vanhest@rotterdam.nl

Received: Dec 052014 | Accepted after revision: March 182016 | First published online: April 212016

Conflict of interest: None declared.

\section{References}

1 Uplekar M, Weil D, Lonnroth K, et al. WHO's new end TB strategy. Lancet 2015; 385: 1799-1801.

2 De Vries G, van Hest RA. From contact investigation to tuberculosis screening of drug addicts and homeless persons in Rotterdam. Eur J Public Health 2006; 16: 133-136.

3 De Vries G, van Hest RA, Richardus JH. Impact of mobile radiographic screening on tuberculosis among drug users and homeless persons. Am J Respir Crit Care Med 2007; 176: 201-207.

4 Golub JE, Dowdy DW. Screening for active tuberculosis: methodological challenges in implementation and evaluation (State of the art series. Active case finding/screening. Number 4 in the series). Int J Tuberc Lung Dis 2013; 17: 856-865.

5 World Health Organization. Systematic screening for active tuberculosis: principles and recommendations. WHO/HTM/TB/2013.04. Geneva, World Health Organization, 2013.

6 Story A, Aldridge RW, Abubakar I, et al. Active case finding for pulmonary tuberculosis using mobile digital chest radiography: an observational study. Int J Tuberc Lung Dis 2012; 16: 1461-1467.

7 Abubakar I, Story A, Lipman M, et al. Diagnostic accuracy of digital chest radiography for pulmonary tuberculosis in a UK urban population. Eur Respir J 2010; 35: 689-692.

8 Van Hest NAH, De Vries G, Smit F, et al. Estimating the coverage of a targeted mobile tuberculosis screening programme among illicit drug users and homeless persons with truncated models. Epidemiol Infect 2008; 136: $628-635$.

9 Story A, Slockers M, Arrazola de Oñate W, et al. Homeless health services, TB and economic crisis: A tale of three cities. Homeless in Europe. www.feantsa.org/spip.php?article133\&lang=en Date last accessed January 29, 2016. Date last updated: 2011.

10 Goetsch U, Bellinger OK, Buettel K-L, et al. Tuberculosis among drug users and homeless persons: impact of voluntary X-ray investigation on active case finding. Infection 2012; 40: 389-395.

11 Bernard C, Sougakoff W, Fournier A, et al. Impact of a 14-year screening programme on tuberculosis transmission among the homeless in Paris. Int J Tuberc Lung Dis 2012; 16: 649-655.

12 Lönnroth K, Migliori GB, Abubakar I, et al. Towards tuberculosis elimination: an action framework for low-incidence countries. Eur Respir J 2015; 45: 928-952.

13 Diel R, Loddenkemper R, Zellweger JP, et al. Old ideas to innovate tuberculosis control: preventive treatment to achieve elimination. Eur Respir J 2013; 42: 785-801.

14 D'Ambrosio L, Dara M, Tadolini M, et al. Tuberculosis elimination: theory and practice in Europe. Eur Respir J 2014; 43: 1410-1420.

15 World Health Organization. Guidelines on the management of latent tuberculosis infection. WHO/HTM/TB/ 2015.01. Geneva, World Health Organization, 2015. 